\title{
Imaging Molecular Structure and Dynamics utilizing X-ray Free-Electron-Laser Sources
}

\author{
Jochen Küpper \\ Center for Free-Electron Laser Science, DESY, Notkestrasse 85, 22607 Hamburg, Germany \\ Department of Physics, Universität Hamburg, Luruper Chaussee 149, 22761 Hamburg, Germany \\ The Hamburg Center for Ultrafast Imaging, Universität Hamburg, Luruper Chaussee 149, 22761 Hamburg, Germany \\ jochen.kuepper@cfel.de, http://www.controlled-molecule-imaging.org
}

\begin{abstract}
Imaging controlled molecules with ultrashort $\mathrm{x}$ ray pulses from free-electron lasers enables the recording of "molecular movies", i.e., snapshots of molecules at work, with spatial (picometer) and temporal (femtosecond) atomic resolution.
\end{abstract}

Hard-x-ray free-electron lasers (FELs) provide femtosecond-duration pulses of x-rays with unprecedented brilliance [1], [2]. These enable the study of ultrafast chemical dynamics of isolated molecules in the gas phase using diffractive-imaging methods [3]-[7]. We exploit various imaging approaches to understand the intrinsic molecular structure and function, which is at the very heart of the chemical and molecular sciences.

Experiments that allow for the creation of structurally pure samples and, subsequently, for the investigation of their intrinsic molecular dynamics and chemical function have developed tremendously over the last few decades, although "there's plenty of room at the bottom" - for better control as well as for further applications. We detail the use of inhomogeneous electric fields for the manipulation of neutral molecules in the gas-phase, i.e., for the separation of complex molecules according to size, structural isomer, and quantum state [8][12]. These quantum-state-selected samples allow for very strong degrees of alignment and orientation [9], [13]-[17]. The produced ensembles of structurally sorted and fixed-inspace molecules are well-suited for imaging experiments, as the availability of many identical molecules in the camera's frame of reference allows for direct, experimental averaging of the recorded signal until it is above noise.

We have performed a number of imaging experiments at the Linac Coherent Light Source (LCLS) at SLAC [2] and the Free-Electron Laser in Hamburg (FLASH) at DESY [18]. These include the direct $\mathrm{x}$-ray-diffractive imaging of aligned isolated gas-phase molecules [19], [20] and photoelectronholography approaches, which are implemented as imaging of molecular-frame photoelectron angular distributions (MFPAD) [21]-[23]. In these first benchmark imaging experiments, we have exploited cold molecular beams from state-of-theart pulsed valves. These beams were further purified using the electric deflector [24], which spatially disperses the beam according to the molecules' quantum states and separates the molecules from the atomic seed gas. The quantum-state selected samples were laser aligned or mixed-field oriented using nanosecond-pulsed Nd:YAG lasers or stretched pulses from amplified Ti:Sapphire laser systems. The latter allows to generate strong alignment and orientation at full FEL repetition rates [15], [25].

Here, we report on two approaches to image these con-

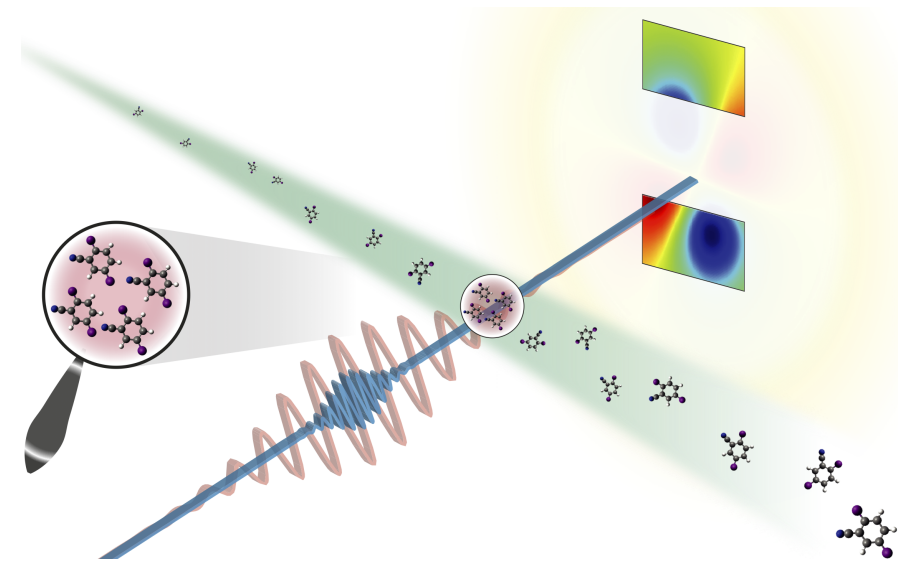

Fig. 1. Sketch of the experimental setup for the atomically-resolved imaging of controlled isolated gas-phase molecules [19].

trolled samples: first, we observe the wide-angle scattering patterns from these molecules. The images recorded on pixelated detectors are the incoherent sums of the coherent-diffractiveimaging (CDI) patterns of the (identical) isolated gas-phase molecules [6], [19], [20]. For the controlled samples employed, this corresponds to a single-molecule pattern with an enhanced signal-to-noise ratio. Alternatively, we have observed the MFPADs following photoionization of these samples. These MFPADs show holographic structures due to the interference of the direct and the scattered parts of the outgoing-electron wave. Especially in the case of core excitations above an absorption edge, e.g., $\mathrm{F}(1 s)$ photoionization, this hologram provides chemical sensitivity for probing of the local structure around the ionized atom [21]-[23]. These experiments will be compared to modern imaging experiments utilizing tabletop laser systems, such as MFPAD measurements following strong-field ionization [26], [27], laser-induced electron diffraction [28], or high-harmonic spectroscopy [29].

Moreover, coincidence ion imaging under similar conditions allows to investigate the ultrafast electronic dynamics of $\mathrm{x}$-ray photoionized dissociating molecules [30], [31].

We summarize current experiments and analyze the feasibility of atomically resolved imaging of ultrafast molecular dynamics. Based on experience from these first experiments, design criteria and experimental approaches for x-ray diffractive imaging of isolated gas-phase molecules are discussed [32]. We propose that these experiments can be performed as secondary, parasitic experiments, upstream of and in-line with more $\mathrm{x}$-ray-intensity demanding primary experiments. 


\section{ACKNOWLEDGMENT}

The author gratefully acknowledges helpful discussions with Stephan Stern, Sebastian Trippel, Daniel Rolles, Artem Rudenko, and Henry Chapman, as well as the many important contributions of his colleagues at the Center for Free-Electron Laser Science, especially in the CFEL Controlled Molecule Imaging Group, as well as the support by many local, national, and international collaborators.

Besides DESY, this work has been supported by the European Research Council through the Consolidator Grant 614507-COMOTION, the Helmholtz Association "Initiative and Networking Fund", and the Deutsche Forschungsgemeinschaft (DFG) through the excellence cluster "The Hamburg Center for Ultrafast Imaging - Structure, Dynamics and Control of Matter at the Atomic Scale" (CUI, DFG-EXC1074).

\section{REFERENCES}

[1] M. Altarelli et al., "The Technical Design Report of the European XFEL,” DESY, Hamburg, Germany, Tech. Rep., Jun 2007. [Online]. Available: http://dx.doi.org/10.3204/DESY_06-097

[2] P. Emma et al., "First lasing and operation of an angstromwavelength free-electron laser," Nat. Photon., vol. 4, pp. 641-647, Jul 2010. [Online]. Available: http://www.nature.com/nphoton/journal/ vaop/ncurrent/abs/nphoton.2010.176.html

[3] R. Neutze, R. Wouts, D. van der Spoel, E. Weckert, and J. Hajdu, "Potential for biomolecular imaging with femtosecond X-ray pulses," Nature, vol. 406, no. 6797, pp. 752-757, Aug. 2000. [Online]. Available: http://dx.doi.org/10.1038/35021099

[4] F. Krasniqi, B. Najjari, L. Strüder, D. Rolles, A. Voitkiv, and J. Ullrich, "Imaging molecules from within: Ultrafast angström-scale structure determination of molecules via photoelectron holography using freeelectron lasers," Phys. Rev. A, vol. 81, p. 033411, 2010.

[5] F. Filsinger, G. Meijer, H. Stapelfeldt, H. Chapman, and J. Küpper, "State- and conformer-selected beams of aligned and oriented molecules for ultrafast diffraction studies," Phys. Chem. Chem. Phys., vol. 13, no. 6, pp. 2076-2087, 2011.

[6] A. Barty, J. Küpper, and H. N. Chapman, "Molecular imaging using x-ray free-electron lasers," Annu. Rev. Phys. Chem., vol. 64, no. 1, pp. 415-435, Apr. 2013. [Online]. Available: http://dx.doi.org/10.1146/ annurev-physchem-032511-143708

[7] J. C. H. Spence and H. N. Chapman, "The birth of a new field," Phil. Trans. R. Soc. B, vol. 369, no. 1647, pp. $20130309-20130309$, Jul 2014.

[8] F. Filsinger, U. Erlekam, G. von Helden, J. Küpper, and G. Meijer, "Selector for structural isomers of neutral molecules," Phys. Rev. Lett., vol. 100, p. 133003, 2008. [Online]. Available: http://dx.doi.org/10. 1103/PhysRevLett.100.133003

[9] L. Holmegaard, J. H. Nielsen, I. Nevo, H. Stapelfeldt, F. Filsinger, J. Küpper, and G. Meijer, "Laser-induced alignment and orientation of quantum-state-selected large molecules," Phys. Rev. Lett., vol. 102, p. 023001, 2009. [Online]. Available: http://dx.doi.org/10.1103/ PhysRevLett.102.023001

[10] F. Filsinger, J. Küpper, G. Meijer, J. L. Hansen, J. Maurer, J. H. Nielsen, L. Holmegaard, and H. Stapelfeldt, "Pure samples of individual conformers: the separation of stereo-isomers of complex molecules using electric fields," Angew. Chem. Int. Ed., vol. 48, pp 6900-6902, 2009. [Online]. Available: http://onlinelibrary.wiley.com/ doi/10.1002/anie.200902650/abstract

[11] J. H. Nielsen, P. Simesen, C. Z. Bisgaard, H. Stapelfeldt, F. Filsinger, B. Friedrich, G. Meijer, and J. Küpper, "Stark-selected beam of groundstate OCS molecules characterized by revivals of impulsive alignment," Phys. Chem. Chem. Phys., vol. 13, pp. 18 971-18 975, 2011.

[12] S. Trippel, Y.-P. Chang, S. Stern, T. Mullins, L. Holmegaard, and J. Küpper, "Spatial separation of state- and size-selected neutral clusters," Phys. Rev. A, vol. 86, p. 033202, Sep. 2012. [Online]. Available: http://pra.aps.org/abstract/PRA/v86/i3/e033202
[13] H. Stapelfeldt and T. Seideman, "Colloquium: Aligning molecules with strong laser pulses," Rev. Mod. Phys., vol. 75, no. 2, pp. 543-557, 2003. [Online]. Available: http://link.aps.org/abstract/RMP/v75/p543

[14] I. Nevo, L. Holmegaard, J. H. Nielsen, J. L. Hansen, H. Stapelfeldt, F. Filsinger, G. Meijer, and J. Küpper, "Laser-induced 3D alignment and orientation of quantum state-selected molecules," Phys. Chem. Chem. Phys., vol. 11, pp. 9912-9918, 2009.

[15] S. Trippel, T. Mullins, N. L. M. Müller, J. S. Kienitz, K. Długołęcki, and J. Küpper, "Strongly aligned and oriented molecular samples at a khz repetition rate," Mol. Phys., vol. 111, p. 1738, 2013.

[16] S. Trippel, T. Mullins, N. L. M. Müller, J. S. Kienitz, J. J. Omiste, H. Stapelfeldt, R. González-Férez, and J. Küpper, "Strongly driven quantum pendulum of the carbonyl sulfide molecule," Phys. Rev. A, vol. 89, p. 051401(R), 2014.

[17] S. Trippel, T. Mullins, N. L. M. Müller, J. S. Kienitz, R. González-Férez, and J. Küpper, "Two-state wave packet for strong field-free molecular orientation," Phys. Rev. Lett., vol. 114, p. 103003, 2015.

[18] V. Ayvazyan et al., "Generation of gw radiation pulses from a vuv free-electron laser operating in the femtosecond regime," Phys. Rev. Lett., vol. 88, p. 104802, Feb 2002. [Online]. Available: http://link.aps.org/doi/10.1103/PhysRevLett.88.104802

[19] J. Küpper et al., "X-ray diffraction from isolated and strongly aligned gas-phase molecules with a free-electron laser," Phys. Rev. Lett., vol. 112, p. 083002, 2014.

[20] S. Stern et al., "Toward atomic resolution diffractive imaging of isolated molecules with x-ray free-electron lasers," Faraday Disc., vol. 171, p. 393, 2014.

[21] R. Boll et al., "Femtosecond photoelectron diffraction on laser-aligned molecules: Towards time-resolved imaging of molecular structure," Phys. Rev. A, vol. 88, p. 061402(R), 2013.

[22] R. Boll et al., "Imaging molecular structure through femtosecond photoelectron diffraction on aligned and oriented gas-phase molecules," Faraday Disc., vol. 171, 2014.

[23] D. Rolles et al., "Femtosecond x-ray photoelectron diffraction on gasphase dibromobenzene molecules," J. Phys. B, vol. 47, no. 12, p. 124035, 2014

[24] F. Filsinger, J. Küpper, G. Meijer, L. Holmegaard, J. H. Nielsen, I. Nevo, J. L. Hansen, and H. Stapelfeldt, "Quantum-state selection, alignment, and orientation of large molecules using static electric and laser fields," J. Chem. Phys., vol. 131, p. 064309, 2009. [Online]. Available: http://scitation.aip.org/content/aip/journal/jcp/131/6/10.1063/1.3194287

[25] T. Kierspel, J. Küpper et al., "Strongly aligned molecules at freeelectron lasers," J. Phys. B, 2015, in preparation for special issue on "science at free-electron lasers".

[26] C. Z. Bisgaard, O. J. Clarkin, G. Wu, A. M. D. Lee, O. Geßner, C. C. Hayden, and A. Stolow, "Time-resolved molecular frame dynamics of fixed-in-space $\mathrm{CS}_{2}$ molecules," Science, vol. 323, no. 5920, pp. 14641468, 2009.

[27] L. Holmegaard et al., "Photoelectron angular distributions from strongfield ionization of oriented molecules," Nat. Phys., vol. 6, p. 428, 2010.

[28] C. I. Blaga, J. Xu, A. D. DiChiara, E. Sistrunk, K. Zhang, P. Agostini, T. A. Miller, L. F. DiMauro, and C. D. Lin, "Imaging ultrafast molecular dynamics with laser-induced electron diffraction," Nature, vol. 483, no. 7388, pp. 194-197, Mar. 2012.

[29] J. Itatani, J. Levesque, D. Zeidler, H. Niikura, H. Pépin, J. C. Kieffer, P. B. Corkum, and D. M. Villeneuve, "Tomographic imaging of molecular orbitals," Nature, vol. 432, pp. 867-871, 2004.

[30] B. Erk et al., "Ultrafast charge rearrangement and nuclear dynamics upon inner-shell multiple ionization of small polyatomic molecules," Phys. Rev. Lett., vol. 110, no. 5, p. 053003, Jan. 2013. [Online]. Available: http://prl.aps.org/abstract/PRL/v110/i5/e053003

[31] B. Erk et al., "Imaging charge transfer in iodomethane upon x-ray photoabsorption." Science, vol. 345, no. 6194, pp. 288-291, Jul. 2014.

[32] S. Stern, H. Chapman, and J. Küpper, "Diffractive imaging of isolated molecules at x-ray free-electron lasers," J. Phys. B, 2015, in preparation for special issue on "science at free-electron lasers". 